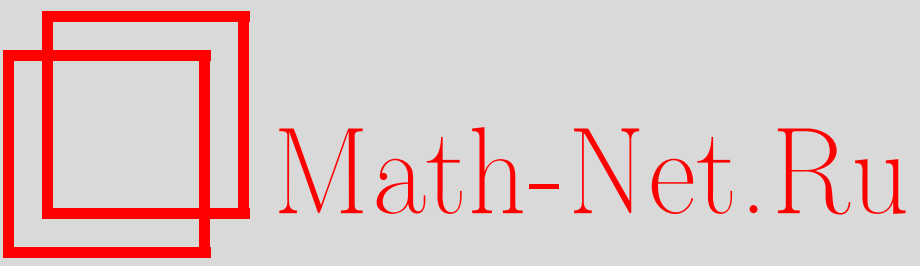

И. А. Вернигора, Ю. Г. Рудой, Расширение лоренцевской симметрии до конформной в пределе сверхвысоких энергий, Вестн. Сам. гос. техн. ун-та. Сер. Физ.-мат. науки, 2011, выпуск 2(), 149-154

DOI: https://doi.org/10.14498/vsgtu905

Использование Общероссийского математического портала Math-Net.Ru подразумевает, что вы прочитали и согласны с пользовательским соглашением

http://www. mathnet.ru/rus/agreement

Параметры загрузки:

IP : 34.239 .49 .27

26 апреля 2023 г., 13:49:24 
УДК 530.18+524.1

\section{РАСШИРЕНИЕ ЛОРЕНЦЕВОЙ СИММЕТРИИ ДО КОНФОРМНОЙ В ПРЕДЕЛЕ СВЕРХВЫСОКИХ ЭНЕРГИЙ}

\section{И. А. Вернигора, Ю.Г. Рудой}

Российский университет дружбы народов, 117198, Москва, ул. Миклухо-Маклая, 6.

E-mails: rudikar@mail.ru, wenera83@mail.ru

Предложено теоретико-групповое обоснование оригинального подхода Киржнииа и Чечина, позволяющего первичным протонам космических лучей сверхвысоких энергий преодолеть энергетический предел Грейзена-Зацепина-Кузвмина (около 50 ЕэВ) в рамках обычных представлений о физической природе внегалактических источников. Показано, что явный вид множителя, деформирующего лоренцев инвариант в пространстве энергий-импульсов, может быть установлен на основе приближенного перехода от лоренцевой симметрии к конформной при значениях лоренц-бактора порядка $10^{10} \div 10^{11}$.

Ключевые слова: группа Лоренща, конформная группа, космические лучи, предел Грейзена-Зачепина-Кузъмина, сверхвысокие энергии.

Введение. Общая характеристика проблемы ГЗК. Вот уже более полувека в астрофизике космических учей (КЛ) в области сверхвысоких энергий от экза- $\left(\mathrm{EэВ}=10^{18}\right.$ эВ) до зетаэлектронвольт $\left(\right.$ ЗэВ $\left.=10^{21} э \mathrm{~B}\right)$ остается нерешенной проблема существования предела Грейзена-Зацепина-Кузьмина $(\Gamma 3 \mathrm{~K})$, теоретически установленного в рамках стандартной релятивистской астрофизики одновременно и независимо в работах [1] и [2]. Согласно этим работам, распределение по энергиям протонной компоненты первичных КЛ должно резко обрываться в области энергий порядка $E_{\Gamma з К ~} \approx 5 \cdot 10^{19}$ эВ за счёт потерь энергии протонов на фоторождение пионов в реакциях вида $p+\gamma \rightarrow p+\pi^{0}$ или $p+\gamma \rightarrow n+\pi^{+}$(с последующим $\beta$-распадом $n \rightarrow p+e^{-}+\nu_{e}$ ). Здесь $\gamma$ - «мягкие» ИК-фотоны реликтового космического излучения, которые в системе покоя протонов первичных КЛ соответствуют жесткому гаммаизлучению.

Однако экспериментальный статус предела ГЗК все еще остается не вполне определенным (см. $[4,5]$, а также обзоры $[7,8])$. Это связано как с недостаточной статистикой событий в области энергий $E_{\Gamma з к} \approx 50$ ЕэВ, так и с трудностью их идентификации. Имеются, однако, серьезные основания полагать, что транс-ГЗК-события все же имеют место, поэтому остается актуальной проблема теоретического обоснования подобной возможности.

Расчёты, проведённые в [1] и [2] (см. также [3]), показывают, что протоны имеющие энергию покоя $\mathrm{E}_{0} \approx 1$ ГэВ, а в лабораторной системе отсчёта -

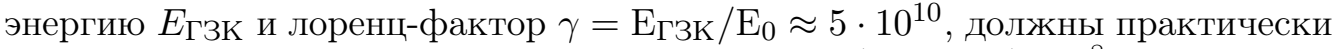
полностью потерять свою энергию за время $\tau \approx(1,0 \div 1,5) \cdot 10^{8}$ лет, проходя при этом расстояние от источника, не превышающее $l \approx c \tau=30 \div 50$ Мпк.

Отсюда следует, что источник протонов столь высоких энергий («зеватрон») должен быть «галактическим», т. е. находиться внутри Местного сверх-

Ирина Александровна Вернигора, аспирант, каф. теоретической физики. Юрий Григоръевич Рудой (д.ф.-м.н., проф.), профессор, каф. теоретической физики. 
скопления галактик. Согласно диаграмме Хилласа (см. [4,5]), таким источником могли бы быть «горячие пятна» радиогалактик - например, М87-Вирго или М82, расположенных на расстоянии порядка $15 \div 25$ Мпк от Земли, а также остатки сверх- или гиперновых и/или гамма-всплески (см. [6]).

Очевидно, что регистрируемый посредством наземных ШАЛ поток протонов от такого рода «единичных» внутригалактических источников должен быть существенно анизотропным, однако имеющиеся наблюдения такому выводу противоречат $[4,5]$. Поэтому реальными кандидатами на роль «зеватронов» являются внегалактические источники, например, активные ядра галактик, но тогда протонам КЛ пришлось бы пройти расстояния не менее 150 Мпк. Последнее явным образом превышает предполагаемый ГЗК-предел, так что возникает (по крайней мере теоретически) проблема преодоления этого предела.

Идея Киржница-Чечина по преодолению предела ГЗК. Попытки подобного преодоления можно разделить на две группы. Одна из них, называемая подходом «новой физики» (см., например, [9]), рассматривает возможности внутригалактического рождения изотропно распределенных транс-ГЗК-протонов в процессах распада некоторых реликтовых объектов.

Однако значительно ранее, начиная с работы Киржница и Чечина [10] (см. также Коулмен и Глэшоу [11] и более поздние работы [12-14]), рассматривалась и другая - на наш взгляд, более реалистическая - возможность: оставаясь в рамках «обычной физики» найти ресурсы её «деформации», в принципе допускающие преодоление предела ГЗК. К сожалению, пионерская работа [10] была впервые замечена и процитирована в зарубежной литературе лишь спустя четверть века в [12]. Согласно [10,11], подобную роль могло бы сыграть отклонение от лоренцевской кинематики при столь высоких значениях лоренц-фактора $\gamma$.

В [10] этот подход был сформулирован лишь на полуфеноменологическом уровне, поэтому целью данной работы является более строгое теоретикогрупповое обоснование подхода Киржница и Чечина [10] и проведение на этой основе численных оценок преодоления ГЗК-предела. Исходная идея состоит в том, что при сверхвысоких значениях энергии и лоренц-фактора $\gamma$ в кинематике любых массивных частиц появляются «конформные» поправки по степеням $1 / \gamma$ (отсутствующие для безмассовых частиц в пределе $1 / \gamma=0$ ).

Выражение для времени прохождения протонов. Согласно [1-3], время прохождения протонов со стартовой энергией $E$ в ЛСО даётся выражением

$$
\tau^{-1}(E)=A \int_{\varepsilon_{\text {пор }}^{\prime} / 2 \gamma}^{\infty} d \varepsilon n_{\gamma}(\varepsilon) \int_{\varepsilon_{\text {пор }}^{\prime}}^{2 \gamma \varepsilon} d \varepsilon^{\prime} \varepsilon^{\prime} \sigma\left(\varepsilon^{\prime}\right) K\left(\varepsilon^{\prime}\right) .
$$

Здесь $A$ - размерная постоянная; $n_{\gamma}(\varepsilon)$ - равновесное планковское распределение (с температурой $T=2,7 \mathrm{~K}$ ) фотонов реликтового излучения с энергией $\varepsilon$ и концентрацией $\approx 400 \mathrm{~cm}^{-3} ; 0 \leqslant \varepsilon^{\prime} \leqslant 2 \gamma \varepsilon ; \gamma=E / E_{0}$-лоренцфактор перехода из ССО протона в СЦИ протона и фотона; $\sigma\left(\varepsilon^{\prime}\right)$-сечение фоторождения пионов, $K\left(\varepsilon^{\prime}\right)$ - коэффициент неупругих потерь энергии протона, имеющий вид $K\left(\varepsilon^{\prime}\right)=(1 / 2)\left\{1-\varphi\left(\varepsilon^{\prime}\right)\right\}=(1 / 2)\left\{1-\Delta / S\left(\varepsilon^{\prime}\right)\right\} ; \Delta=$ $=M^{2}-m^{2}=$ const $>0 ; M, m-$ массы протона и пиона, $m=0,14 M ; S\left(\varepsilon^{\prime}\right)=$ $=M^{2}+2 M \varepsilon^{\prime}$-лоренц-инвариант; $\varepsilon_{\text {пор }}^{\prime}=m(1+m M / 2) \approx 1,07 m-$ пороговая 
энергия рождения пиона, причём $K\left(\varepsilon_{\text {пор }}^{\prime}\right) \approx 0,12$. Очевидно, что вид входящих в (1) функций $n_{\gamma}(\varepsilon)$ и $\sigma\left(\varepsilon^{\prime}\right)$ задан однозначно, и только функцию $K\left(\varepsilon^{\prime}\right)$ (а следовательно, и величину $\tau^{-1}(E)$ ) можно в принципе изменить посредством изменения лоренц-инварианта $S\left(\varepsilon^{\prime}\right)$.

Подходы к деформации лоренцевской кинематики. Именно эта логика лежит в основе подхода Киржница и Чечина [10], стартующего с лоренц- $(L)$ инварианта в пространстве энергий и импульсов:

$$
I_{L}\left(p ; E_{0}\right)=E^{2}-p^{2}=I_{L}\left(0 ; E_{0}\right)=E_{0}^{2} ; \quad E_{L}\left(p ; E_{0}\right)=\left(E_{0}^{2}+p^{2}\right)^{1 / 2},
$$

где $E_{L}\left(p ; E_{0}\right)$-лоренцев закон дисперсии. Для величин $\beta \equiv v_{L}$ (скорости частицы) и лоренц-фактора $\gamma$ имеем (при $c=1)$ :

$$
\begin{aligned}
& \mathbf{v}_{L}\left(p ; E_{0}\right)=\partial E_{L}\left(p ; E_{0}\right) / \partial \mathbf{p}=\mathbf{p} / E_{L}\left(p ; E_{0}\right), \quad v_{L}\left(p ; E_{0}\right) \equiv\left|\mathbf{v}_{L}\left(p ; E_{0}\right)\right| \leqslant 1 \\
& \gamma\left(p ; E_{0}\right) \equiv E_{L}\left(p ; E_{0}\right) / E_{0} \geqslant 1,1 / \gamma^{2}\left(p ; E_{0}\right)=1-v_{L}^{2}\left(p ; E_{0}\right), 0 \leqslant 1 / \gamma\left(p ; E_{0}\right) \leqslant 1 .
\end{aligned}
$$

В контексте данной работы особый интерес представляет предельный случай безмассовой частицы $\left(E_{0}=0\right)$, для которого

$$
I_{L}(p ; 0)=0, \quad E_{L}(p ; 0)=p, \quad v_{L}(p ; 0)=1, \quad 1 / \gamma(p ; 0)=0 .
$$

Феноменологический подход Киржница и Чечина [10]. Поскольку лоренцева кинематика (2)-(5) приводит к пределу ГЗК для (1), Киржниц и Чечин [10] конструируют вместо (2) новое выражение для инварианта $I\left(p ; E_{0}\right)-$ и, следовательно, для закона дисперсии $E\left(p ; E_{0}\right)$. С этой целью выражение для $I_{L}\left(p ; E_{0}\right)$ умножается в [10] на скалярный множитель $f\left(p ; E_{0}\right)$, который определяется на основе чисто феноменологических соображений.

В частности, поскольку согласно $(2)$ и $(3) I_{L}\left(p ; E_{0}\right)=E^{2}\left[1-v_{L}^{2}\left(p ; E_{0}\right)\right]$, авторы полагают естественным (в рамках идеи о «минимальной» деформации) искать $f\left(p ; E_{0}\right)$ в виде $f\left(v_{L}^{2}\left(p ; E_{0}\right)\right)$, что с учётом $(4)$ равносильно $f\left(\gamma^{2}\left(p ; E_{0}\right)\right)$. В [10] предполагается, что в безмассовом пределе $\gamma^{2} \rightarrow \infty$ (и, соответственно, $v_{L} \rightarrow 1$ ) функция $f\left(\gamma^{2}\right)$ имеет конечный предел $f(\infty)$ (вообще говоря, отличный от 1); очевидно, такое предположение не влияет на закон дисперсии безмассовых частиц, поскольку для них $I(p ; 0)=f(\infty) I_{L}(p ; 0)=0$, где учтено свойство (5).

Окончательно Киржниц и Чечин [10] получают для функции $f\left(\gamma^{2}\right)$ выражение (формула (19) в работе [10]):

$$
f\left(\gamma^{2}\right) \approx 1+|\alpha| \gamma^{4}, \quad|\alpha| \gamma^{4} \leqslant 1, \quad|\alpha| \approx 10^{-44}, \quad \gamma \approx \gamma_{0} \equiv 10^{11} .
$$

Основная идея данной работы состоит в построении уточненного выражения для «деформирующего» множителя $f\left(\gamma^{2}\right)$, который мы будем называть конформным множителем и обозначать $C\left(\xi^{2}\right)$, где величина $\xi \equiv \gamma^{-1}$ рассматривается как малый параметр. Для удобства сравнения результатов нашей работы с работой [10] мы будем обозначать функцию $C\left(\xi^{2}\right)$ как $g\left(\gamma^{2}\right)$.

Оставаясь в целом в рамках логики работы [10], мы предлагаем дополнительную идею, состоящую не просто в некотором феноменологическом «нарушении» группы преобразований Лоренца-Пуанкаре, а в её расширении до 
хорошо известной конформной группы Вейля-Фока. Для этого следует заменить лоренцев скаляр $I_{L}\left(p ; E_{0}\right)$, инвариантный при любых значениях $E_{0}$ и $\xi$, на конформный скаляр $I_{C}\left(p ; E_{0}\right)=C(\xi) I_{L}\left(p ; E_{0}\right)$, который является точным инвариантом только в безмассовом случае ${ }^{1}: E_{0}=0, \xi=\gamma^{-1}=0$, когда в соответствии с $(5)$ имеем $I_{C}(p ; 0)=C(\xi) I_{L}(p ; 0)=0$. Именно это обстоятельство указывает естественный путь построения теории возмущений для $C(\xi)$ по малому параметру $\xi=\gamma^{-1} \ll 1$.

Теоретико-групповой подход. Наиболее удобным и естественным инструментом такого подхода являются преобразования конформной группы, причём для наших целей достаточно ограничиться лишь преобразованиями Мёбиуса $C_{4}(c)$, известными как «специальные конформные» преобразования.

Под действием этих преобразований любой 4-вектор $P$ переходит в $P_{C}=$ $=\mathbf{C}(P ; c) P$, где матрица $\mathbf{C}$ зависит от $P$, и, кроме того, определяется произвольным 4-вектором $c$. Преобразование $\mathbf{C}(P ; c)$ имеет явный вид:

$$
P_{C}=C(P ; c) P+\Delta P(P ; c), \quad \Delta P(P ; c)=C(P ; c) c P^{2}, \quad P_{C}^{2}=C(P ; c) P^{2}
$$

и является нелинейным, неоднородным и анизотропным масштабным преобразованием, причем конформный множитель $C(P ; c)$ даётся выражением

$$
C(P ; c)=[\sigma(P ; c)]^{-1}, \quad \sigma(P ; c)=1-2 c P+c^{2} P^{2}=c^{2}\left(P-c / c^{2}\right)^{2} .
$$

Как видно из $(7)$, где вообще $C \neq 1$, лоренц-инвариантная величина $P^{2}=E_{0}^{2}$ не всегда является конформным инвариантом; исключением является $P^{2}=0$ (при $E_{0}=0$ и $\left.\xi=0\right)$. Физически ясно, что для ненулевых, но малых значений $E_{0}$ и $\xi \ll 1$ конформный множитель $C(P ; c)$ при $\xi \ll 1$ мало отличается от $C(0 ; c)=$ const.

Перейдём от 4-векторов энергии-импульса $P(E, \mathbf{p})$ к их безразмерным или однородным аналогам $V(E, \mathbf{p}) \equiv P(E, \mathbf{p}) / E=(1, \mathbf{p} / E)$. Тогда согласно кинематике (2)-(4) вектор $V_{L}(E, \mathbf{p})=\left(1, \mathbf{v}_{L}\left(p ; E_{0}\right)\right)$ имеет смысл 4-вектора скорости, причём $V_{L}^{2} \equiv 1 / \gamma^{2},\left(V_{L}^{\text {св }}\right)^{2}=0$, так что лоренц- $(L)$-инвариантом является величина $\gamma^{2} V_{L}^{2} \equiv 1$.

Численные оценки возможного преодоления предела ГЗК. Ограничим далее выбор вектора $c$ в $C(V, c)$ выделенным направлением $A V$, где $A=-a \gamma^{*}$, $a>0$ - численный множитель порядка $1 \div 10^{2}, \gamma^{*} \equiv E^{*} / E_{0} \gg 1$ - характеристическое значение лоренц-фактора; например, для $E_{0} \approx 1$ ГэВ целесообразно принять $E^{*}=E_{\text {Планк }} \approx 1,2 \cdot 10^{19}$ ГэВ,$\gamma^{*} \simeq 10^{19}$,так что $\gamma^{*} / \gamma_{\text {ГзК }} \approx 10^{8} \div 10^{9}$.

В результате конформный множитель $C(V, A V)=\left(1-A V^{2}\right)^{-1} \leqslant 1$ принимает вид

$$
g\left(\gamma^{2}\right)=\left(1+a \gamma^{*} \gamma^{-2}\right) \approx 1-a \gamma^{*} \gamma^{-2},
$$

в ряде отношений отличный от выражения (6), полученного Киржницем и Чечиным [10]: прежде всего, как и должно быть, $g\left(\gamma^{2}\right) \leqslant 1$, тогда как $f\left(\gamma^{2}\right) \geqslant 1$. Кроме того, $g\left(\gamma^{2}\right)$ имеет точку перегиба и конечный предел $g\left(\gamma^{2}\right) \rightarrow 1$ при $\gamma \rightarrow \infty$, тогда как для получения конечного значения монотонно растущей функции $f\left(\gamma^{2}\right)$ при $\gamma \rightarrow \infty$ требуется добавление в правую часть (6) слагаемых вида $-|\beta| \gamma^{6}$.

\footnotetext{
${ }^{1}$ Здесь предполагается, что величина $C(0)$, по смыслу совпадающая с $f(\infty)$ из [10], отлична от нуля; ниже это находит подтверждение (см. формулу (9)).
} 
В заключение рассмотрим, какое влияние на время прохождения протонов (1) окажет замена входящего в коэффициент неупругости $K$ лоренцева инварианта $S_{L}$ на конформный инвариант $S_{C}=g S_{L}, g \leqslant 1$. Опуская всюду аргумент $\varepsilon$, имеем: $K_{L}=1 / 2(1-\varphi), \varphi=\Delta / S_{L}, \Delta>0, \varphi \leqslant 1$, так что $0 \leqslant K_{L} \leqslant 1$; с другой стороны, $K_{C}=1 / 2(1-\lambda \varphi)$, где $\lambda=S_{L} / S_{C}=(1 / g) \geqslant 1$.

Пусть по астрофизическим соображениям требуется увеличить время и расстояние прохождения протонов с энергией $E_{\Gamma з \mathrm{~K}}$ в $(1 / \eta) \geqslant 1$ раз: $l_{C} / l_{L}=$ $=\tau_{C} / \tau_{L}=1 / \eta$, так что $K_{C} / K_{L}=\eta \leqslant 1$ и $\lambda=(1 / \varphi)[1-\eta(1-\varphi)]$. Учитывая,

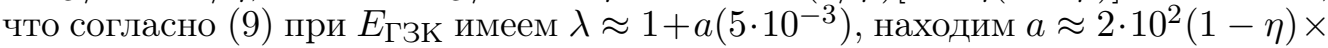
$\times[(1 / \varphi)-1]$. Далее, ограничиваясь значением $K_{L}=0,12$ на пороге фоторождения пионов, находим $\varphi=0,76$, откуда $a \approx 66(1-\eta)$. Для прохождения протонами внегалактического расстояния $\sim 150 \div 200$ Мпк достаточно положить $\eta \approx 0,25$, что даёт $a \approx 50$.

Заключение. В работе показано, что идею пионерской работы Киржница-Чечина [10] о слабом «нарушении» лоренц-инвариантности необходимо дополнить идеей её расширения до конформной инвариантности. Это позволяет при разумных значениях конформных параметров увеличить расстояние, проходимое первичными протонами КЛ сверхвысоких энергий, до $150 \div 200$ Мпк (или более), т. е. преодолеть предел ГЗК (соответствующий значениям лоренц-фактора $\sim 10^{10} \div 10^{11}$ ), если это будет востребовано для описания предстоящих широкомасштабных экспериментов - как на действующих установках «Пьер Оже», HiRes, AGASA, «Якутск», так и на планируемой установке EUSO.

\section{БИБЛИОГРАФИЧЕСКИЙ СПИСОК}

1. Greisen K. End to the cosmic-ray spectrum?// Phys. Rev. Lett., 1966. Vol.16, no. 17. Pp. 748-750.

2. Зацепин Г. Т., Кузъмин В. А. О верхней границе спектра космических лучей // Писъма в ЖКЭТФ, 1966. Т. 4, № 3. С. 114-117; англ. пер.: Zatsepin G. T., Kuz'min V. A. Upper limit of the spectrum of cosmic rays // JETP, 1966. Vol. 4, no. 3. Pp. 78-80.

3. Stecker F. W. Effect of photomeson production by the universal radiation field on the highenergy cosmic rays // Phys. Rev. Lett., 1968. Vol.21, no. 14. Pp. 1016-1018.

4. Панасюк М. И. Странники Вселенной, или эхо Большого взрыва. Фрязино: Век 2, 2005. 267 c. [Panasyuk M.I. Wanderers of the Universe or the Big Bang Echo. Fryazino: Vek 2, 2005. 267 pp.]

5. Засов А.В., Постнов К.А. Общая астрофизика. Фрязино: Век 2, 2006. 496 с. [Zasov A. V., Postnov K. A. General astrophysics. Fryazino: Vek 2, 2006. 496 pp.]

6. Птускин В.С. О происхождении галактических космических лучей// УФН, 2007. T. 177, №5. C. 558-565; англ. пер.: Ptuskin V.S. On the origin of galactic cosmic rays // Phys. Usp., 2007. Vol. 50, no. 5. Pp. 534-540.

7. Иванов А.А., Кнуренко С.П., Правдин И. М., Красилъников А.Д., Слепиов И. Е. Поиск внегалактических источников космических лучей в области предельных энергий // Извест. РАН. Сер. Физическал, 2009. Т. 73, № 5. С. 581-583; англ. пер.: Ivanov A. A., Knurenko S.P., Pravdin M.I., Krasilnikov A.D., Sleptsov I.E. A search for extragalactic sources of cosmic rays in the ultra-high energy domain // Bulletin of the Russian Academy of Sciences: Physics, 2009. Vol.73, no. 5. Pp. 544-546.

8. Olinto A.V., Adams H.J., Dermer C.D. et al. White paper on ultra-high energy cosmic rays: http://uhecr.uchicago.edu.

9. Berezinsky V.S. Ultra-high energy cosmic rays // Nucl. Phys. B., 2000. Vol. 81. Pp. 311-322.

10. Киржнии, Д.А., Чечин В.А. Космические лучи сверхвысоких энергий и возможное обобщение релятивистской теории // Ядерн. физ., 1972. Т. 15, №5. С. 1051-1059. 
[Kirzhnits D. A., Chechin V.A. Ultra-high energy cosmic rays and possible generalization of the relativistic theory // Yadern. Fiz., 1972. Vol.15, no. 5. Pp. 1051-1059].

11. Coleman S., Glashow S. L. High-energy tests of Lorentz invariance// Phys. Rev. D, 1999. Vol. 59, no. 11, 116008. 14 pp.

12. Gonzalez-Mestres L. Deformed Lorentz symmetry and high-energy astrophysics (I), 2000, arXiv: physics/0003080 [physics.gen-ph].

13. Scully S. T., Stecker F. W. Lorentz invariance violation and the observed spectrum of ultrahigh energy cosmic rays // Astroparticle Physics. Vol.31, no.3. Pp. 220-225, arXiv: 0811.2230 [astro-ph].

14. Jacobson T., Liberati S., Mattingly D. Astrophysical bounds on Planck suppressed Lorentz violation // Lect. Not. Phys., 2005. Vol. 669. Pp. 101-130, arXiv: hep-ph/0407370.

MSC: 83B99, 85A99

\section{EXTENSION OF THE LORENTZ SYMMETRY UP TO CONFORMAL IN THE LIMIT OF ULTRAHIGH ENERGIES}

\section{A. Vernigora, Yu. G. Rudoy}

People's Friendship University of Russia, 6, Mikluho-Maklaja st., Moscow, 117198, Russia.

E-mails: rudikar@mail.ru, wenera83@mail.ru

The group-theoretical justification is presented for the original approach by Kirznits and Chechin which allows for the primary protons of ultra-high energy cosmic rays to overcome the energetic limit (about 50 EeV) of Greisen-Zatsepin-Kuzmin remaining in the scope of the usual ideas about the nature of the extra-galactic sources of the cosmic rays. It is shown that the explicit form of the factor deforming the Lorentz invariant in the energy-momentum space may be found on the grounds of the approximate transition from Lorentz symmetry to the conformal values of the Lorentz-factor of the order $10^{10} \div 10^{11}$.

Key words: Lorentz group, conformal group, cosmic rays, Greisen-Zatsepin-Kuzmin limit, ultra-high energy.

Original article submitted 21/XII/2010; revision submitted 04/IV/2011.

Irina A. Vernigora, Postgraduate student, Dept. of Theoretical Physics.

Yuriy G. Rudoy (Dr. Sci. (Phys. \& Math.)), Dept. of Theoretical Physics. 